\title{
Release mechanisms of major DAMPs
}

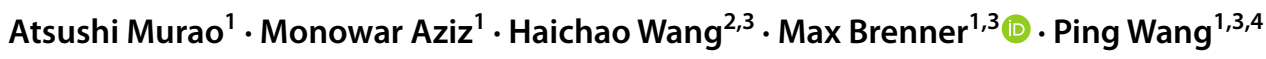

Accepted: 26 February 2021 / Published online: 13 March 2021

(c) The Author(s) 2021

\begin{abstract}
Damage-associated molecular patterns (DAMPs) are endogenous molecules which foment inflammation and are associated with disorders in sepsis and cancer. Thus, therapeutically targeting DAMPs has potential to provide novel and effective treatments. When establishing anti-DAMP strategies, it is important not only to focus on the DAMPs as inflammatory mediators but also to take into account the underlying mechanisms of their release from cells and tissues. DAMPs can be released passively by membrane rupture due to necrosis/necroptosis, although the mechanisms of release appear to differ between the DAMPs. Other types of cell death, such as apoptosis, pyroptosis, ferroptosis and NETosis, can also contribute to DAMP release. In addition, some DAMPs can be exported actively from live cells by exocytosis of secretory lysosomes or exosomes, ectosomes, and activation of cell membrane channel pores. Here we review the shared and DAMP-specific mechanisms reported in the literature for high mobility group box 1, ATP, extracellular cold-inducible RNA-binding protein, histones, heat shock proteins, extracellular RNAs and cell-free DNA.
\end{abstract}

Keywords DAMP $\cdot$ Release $\cdot$ Necrosis $\cdot$ Apoptosis $\cdot$ Exocytosis $\cdot$ Exosome

\section{Introduction}

Damage-associated molecular patterns (DAMPs) are endogenous molecules which serve as potent activators of the immune system [1]. Examples of DAMPs include nuclear and mitochondrial DNA, RNA, nucleotides and nucleosides, DNA-binding molecules, temperature-shock proteins, and uric acid [1-3]. DAMPs normally reside inside the cell playing diverse roles in homeostasis, but are released to

Max Brenner and Ping Wang have contributed equally to this work.

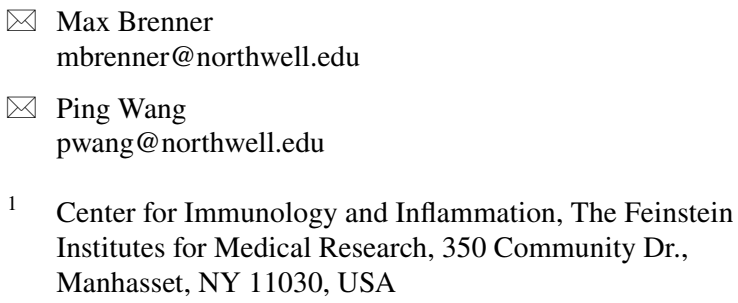

1 Center for Immunology and Inflammation, The Feinstein Institutes for Medical Research, 350 Community Dr., Manhasset, NY 11030, USA

2 Center for Biomedical Science, The Feinstein Institutes for Medical Research, Manhasset, NY, USA

3 Department of Molecular Medicine, Zucker School of Medicine at Hofstra/Northwell, Manhasset, NY, USA

4 Department of Surgery, Zucker School of Medicine at Hofstra/Northwell, Manhasset, NY, USA the extracellular space when cells are exposed to stress [1]. Cellular stressors that can lead to DAMP release include a wide array of physical (trauma, radiation), chemical (toxins, osmolarity), metabolic (ischemia/reperfusion), and infectious (viruses, bacteria, protozoa) factors $[1,4,5]$. Once outside the cell, DAMPs are recognized by other cells via their interaction with cellular receptors such as pattern recognition receptors (PRRs), which then upregulate stress response mechanisms that often converge to form a positive feedback loop of tissue injury and inflammation [1]. Indeed, the relevance of DAMPs to various diseases is supported by a number of studies. In sepsis, circulating DAMPs correlate with disease severity and their inhibition has been shown to improve the outcomes in experimental models of sepsis [1]. During cancer different types of DAMPs can promote tumor establishment and progression as well as metastasis [6]. DAMPs are upregulated systemically and locally in patients with autoimmune diseases such as rheumatoid arthritis and their neutralization has shown to prevent the disease progression in animal models [7]. Therefore, DAMP-inhibiting molecules have the potential to significantly attenuate inflammation and in the future may yield a novel class of anti-inflammatory drugs able to finally treat trauma, ischemia/reperfusion injury, sepsis, neuroinflammation, and other pathophysiological conditions irresponsive 
to existing immune modulatory drugs. Indeed, molecules targeting a number of DAMPs are already in the process of being developed as potential therapeutic agents [8]. At the moment, the predominant strategies to decrease the effects of DAMPs consist of antibody neutralization, competitive antagonism, and enzymatic inactivation [1, 8]. An alternative approach at a more fundamental level is to actively suppress the cellular release of DAMPs. While thorough understanding of the mechanisms of DAMP release may lead to new treatments to attenuate the proinflammatory activity of DAMPs, it might also help to overcome the difficulties we are facing with other forms of immunotherapy such as cytokine removal [9].

Here we review the mechanisms of DAMP release that have been reported. We first summarize universal mechanisms affecting most if not all DAMPs (i.e., different types of cell death, lysosomal- and exosomal-exocytosis) (Fig. 1). We then outline the mechanisms that have been described for selected DAMPs individually, including high mobility group box 1 (HMGB1), ATP, extracellular cold-inducible RNA-binding protein (eCIRP), histones, heat shock proteins (HSPs), extracellular RNAs (exRNAs) and cell-free DNA (cfDNA), as they have been well-studied among other DAMPs (Table 1).

\section{Universal mechanisms of DAMP release}

Different DAMPs share common mechanisms for their release. The release mechanisms can be largely divided into two categories: passive release mainly due to cell death and active release from live cells represented by exocytosis. To be precise, with the exception of necrosis, the other forms of cell death are not entirely passive but rather a regulated process, and some of the mechanisms described under cell death do not always lead to cell death (e.g., NETosis can be suicidal and vital). Thus, it has to be noted that the following categorization is not completely clear-cut and somewhat vague with overlap.

\section{Passive release (cell death)}

DAMPs are well known to be released during different types of cell death. Necrosis is the most common cell death to cause passive release of DAMPs, whereas necroptosis, apoptosis, pyroptosis, ferroptosis and extracellular traps can also contribute to DAMP release. Theoretically, necrosis can cause the release of mixed DAMPs since the cell boundary is lost due to membrane rupture and any cellular components would be released. On the other hand, different forms of cell death can be rather specific to the types of DAMPs as to their release according to the mechanisms. For example, apoptosis leads to the release of nuclear DAMPs following chromatin condensation and DNA fragmentation. Extracellular traps released during NETosis contain DAMPs which are mainly nuclear molecules and antimicrobial enzymes. Conversely, DAMPs can give clues to by which cell death they were released. The fragment length of cfDNA is different according to the type of cell death; cfDNA originating from apoptotic cells is $\sim 180 \mathrm{bp}$ due to the fragmentation, while cfDNA released from necrotic cells can be as long as $>10,000 \mathrm{bp}$ [10]. HMGB1 released by pyroptosis via
Fig. 1 Universal mechanisms of DAMP release. Common mechanisms of DAMP release from the cells are represented by necrosis/necroptosis, apoptosis, pyroptosis, ferroptosis, extracellular traps, secretory lysosomes and exosomes. RIPK receptorinteracting serine/threonineprotein kinase, $M L K L$ mixed lineage kinase domain like pseudokinase, GSDMD gasdermin D, GPX4 glutathione peroxidase $4, R O S$ reactive oxygen species, $P A D 4$ peptidylarginine deiminase 4, Me methylation, $A c$ acetylation, $P$ phosphorylation, $C$ it citrullination

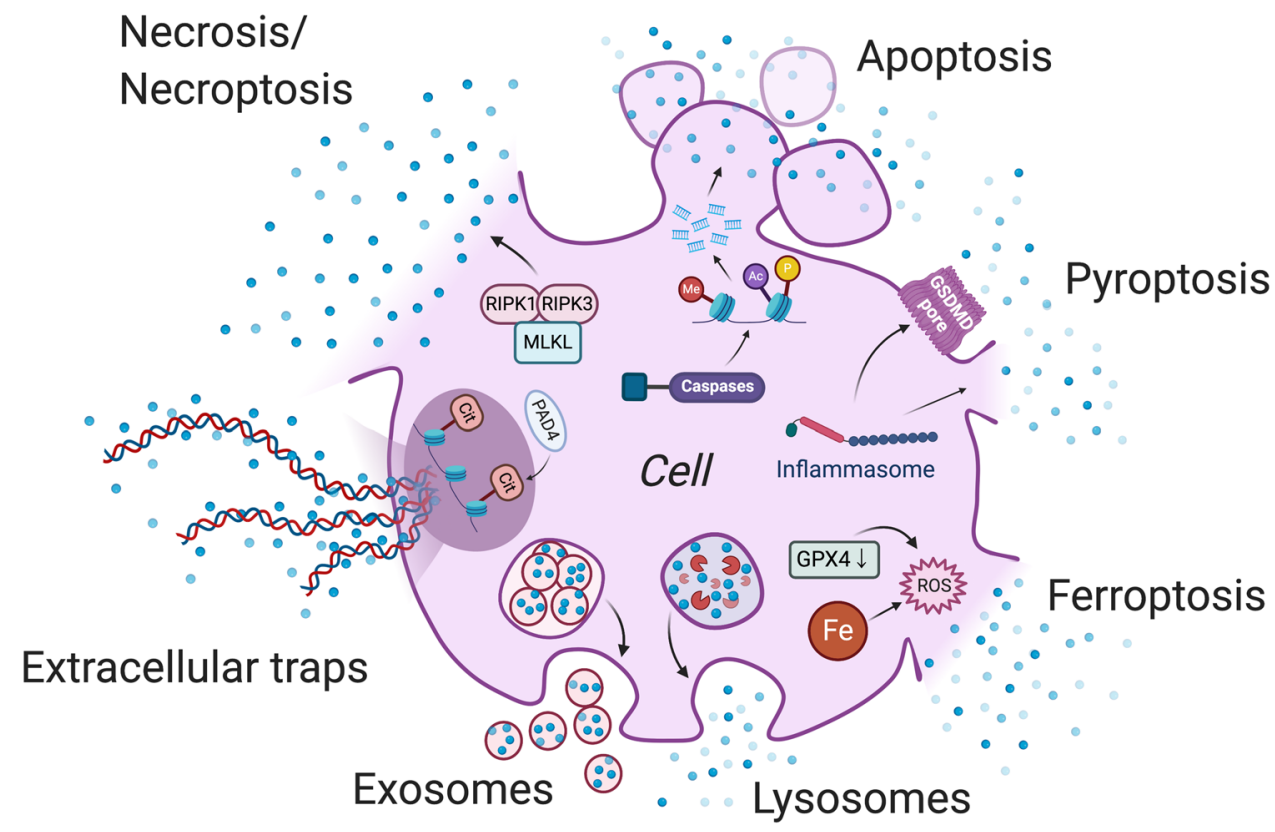




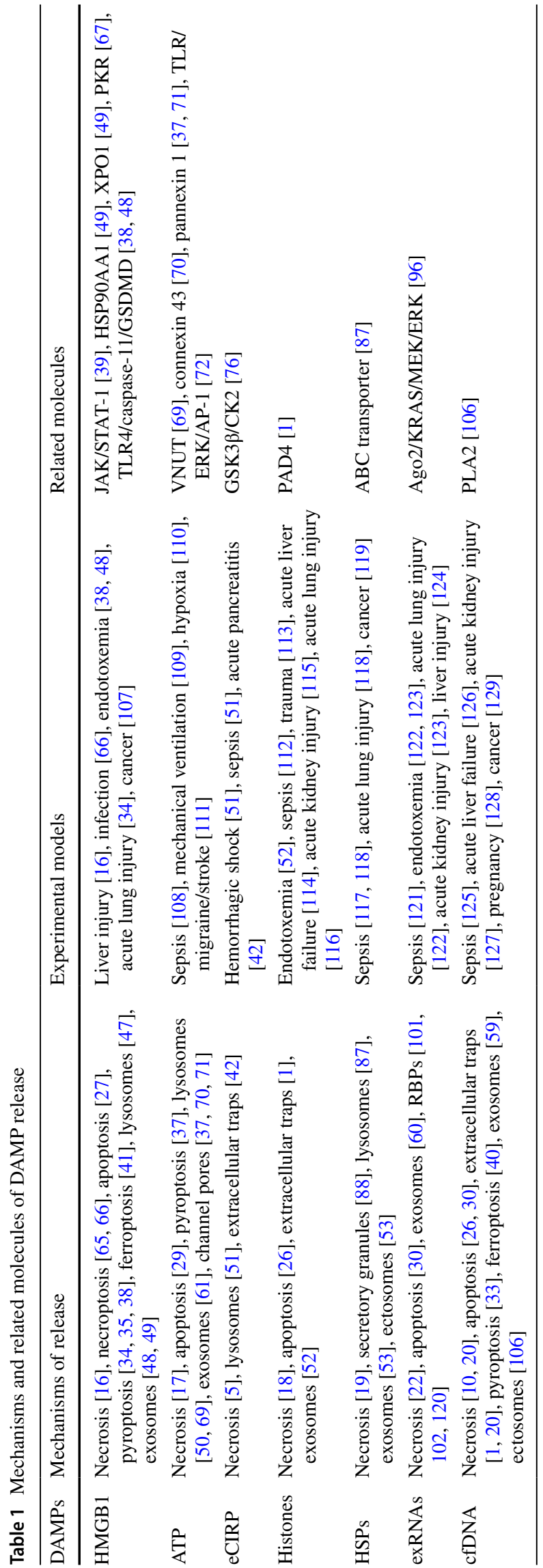

inflammasome pathway is hyperacetylated, which is not seen when it is released from necrotic or apoptotic cells. In addition, the redox state of HMGB1 is in the disulfide form after pyroptosis, in the fully reduced or disulfide form after necrosis, and in the fully oxidized form (sulfonyl HMGB1) after apoptosis [11]. Different DAMPs can be released at the different stages even within the same type of cell death. During apoptosis, ATP is released at the pre-apoptotic stage while HMGB1 is released at the late stage $[12,13]$. Besides the preceding DAMPs, it still largely remains elusive and further studies are awaited as to the types and stages of the cell death of their origin.

\section{Necrosis and necroptosis}

Passive release of DAMPs is typically described to occur as a result of necrosis. Necrosis is commonly caused by extreme chemical or physical insults such as the presence of toxins or trauma, and is characterized by cell swelling and plasma membrane rupture [14]. Tissue ischemia and hypoxia also trigger necrosis by depleting intracellular ATP to unbalance the pump-leak mechanism leading to an influx of $\mathrm{Na}^{+}$ and water, which causes cell swelling. Reperfusion can further damage the cells by inducing the generation of multiple oxidants and free radicals [15]. A number of DAMPs have been found to be released by necrosis including, but not limited to, HMGB1, ATP, histones, HSPs, exRNAs, cfDNA, and possibly eCIRP [1, 3, 5, 10, 16-22].

Although cytoplasmic membrane rupture can be uncontrolled accidental (mechanical or chemical) events, it can also be a regulated process governed by specific caspases and kinases. For instance, necroptosis occurs as a result of receptor-interacting serine/threonine-protein kinase 1 (RIPK1) activation followed by RIPK3-dependent phosphorylation of mixed lineage kinase domain like pseudokinase (MLKL) to induce MLKL oligomerization, which results in plasma membrane rupture [14]. DAMP release has been significantly less studied in the context of necroptosis than of necrosis. However, as membrane integrity is lost in necroptosis in a fashion similar to necrosis, theoretically necroptosis also results in the release of DAMPs and other cellular components to the extracellular space [23].

\section{Apoptosis}

Apoptosis is a regulated cell death without loss of plasma membrane. Morphological features of apoptosis are cytosolic shrinkage, membrane blebbing, chromatin condensation, and DNA fragmentation [14]. The sequential activation of cysteine proteases and endonucleases is the main mechanism of apoptosis, which consists of extrinsic and intrinsic pathway. Extrinsic pathway is induced by caspase-8/caspase-10 or caspase- 9 activated by death receptor- or dependence 
receptor-stimulation, respectively. Intrinsic pathway, on the other hand, is initiated by mitochondrial outer membrane permeabilization followed by the release of mitochondrial proteins, which subsequently causes caspase- 9 activation. Both pathways converge on common effector caspases, i.e., caspase-3, caspase- 6 and caspase-7, to induce apoptosis [24]. In its early stages, apoptosis is traditionally considered a non-immunogenic form of cell death that prevents the release of intracellular contents because there is no loss of membrane integrity. However, recently it has been revealed that apoptosis can be immunogenic under stress conditions such as chemotherapy or physical modalities. This form of apoptosis is named "immunogenic cell death" (ICD) and is characterized by DAMP release [25]. Indeed, apoptosis has been shown to cause nuclear substances to get exposed at the cell surface and released to the extracellular space and thus act as DAMPs [26]. In the line with its mechanism, nuclear molecules, such as HMGB 1, histones, exRNAs and cfDNA as well as ATP have been shown to be released by apoptosis $[1,10,26-30]$. Release mechanisms during apoptosis can be different between DAMPs. Histone release from apoptotic cells is highly associated with DNA fragmentation, which is mediated by caspase-activated DNase/DNA fragmentation factor [28]. ATP has shown to be released from apoptotic cells induced by ER stress in a protein kinase R-like endoplasmic reticulum kinase (PERK) dependent manner [12]. cfDNA and exRNAs have been found in the microparticles released from apoptotic cells [30]. Despite the preceding studies, elucidation of more detailed mechanisms for each DAMP release is awaited in apoptosis.

\section{Pyroptosis}

Another form of caspase-dependent cell death is pyroptosis, which is induced via the activation of caspase- 1 following that of inflammasomes such as NLRP3 or via caspase-4/5/11 activation typically initiated by intracellular LPS [24, 31]. The inflammatory caspase $(-1,-4,-5,-11)$ activation further induces the cleavage of gasdermin D (GSDMD) to promote pore formation in the membrane, allowing the release of intracellular molecules [24, 31]. A recent study has revealed that intracellular protein DDX $3 \mathrm{X}$ promotes NLRP3 inflammasome activation, which can be inhibited by the induction of stress granules causing the sequestration of DDX3X, thus acts as a live-or-die checkpoint in stressed cells [32]. Pyroptosis is typically known for the release of IL-1B, though some other DAMPs such as HMGB1, ATP and cfDNA can be released by cells undergoing this type of cell death [1,33-37]. Even sharing the same signaling pathway, IL-1 $1 \beta$ is well known to be released through GSDMD pore whereas a study has shown HMGB1 was released as a result of cell lysis during pyroptosis [38]. In addition, post-translational modifications are required prior to pyroptosis for the release of HMGB1 as described later [39].

\section{Ferroptosis}

Ferroptosis is a programmed cell death accompanied by iron accumulation and lipid peroxidation. Its morphological features include a loss of membrane integrity, cytoplasmic swelling, swelling of cytoplasmic organelles and moderate chromatin condensation [40]. Intracellular iron accumulation, which can be induced by ferroptosis activators such as erastin and RSL3, causes oxidative stress directly by generating reactive oxygen species (ROS) via the Fenton reaction as well as activating the enzymes responsible for lipid peroxidation and oxygen homeostasis. Glutathione peroxidase 4 (GPX4) is an anti-oxidative enzyme which plays a major role in regulating ferroptosis by preventing lipid peroxidation. Thus, inhibition of GPX4 is a well know mechanism for inducing ferroptosis [40]. Although it is a relatively new concept and less has been elucidated yet, HMGB 1 and cfDNA have been regarded to be released by ferroptosis [ 40 , 41]. A study has shown that HMGB1 release during ferroptosis was due to HMGB1 acetylation induced by histone deacetylase (HDAC) inhibition mediated by autophagy [41].

\section{Extracellular traps}

Neutrophil extracellular traps (NETs) are web-like chromatin-based structures released by neutrophils primarily for pathogen clearance via a regulated process, called NETosis [1]. It is typically described that in the neutrophils undergoing NETosis activated peptidylarginine deiminase 4 (PAD4) citrullinates histones leading to chromatin decondensation accompanied by the dissolution of nuclear and granule membranes. DNA and histones mix with granule-derived antimicrobial peptides in the cytoplasm and are extruded into the extracellular space [1]. Conventionally NETosis was regarded to be a suicidal process leading to cell death (i.e., suicidal NETosis), however later it was found that NETs can also be released from live cells (i.e., vital NETosis) [1]. NETs can be detrimental as they contain not only typical DAMPs, such as histones, cfDNA and eCIRP, but also antimicrobial enzymes, such as neutrophil elastase (NE) and myeloperoxidase (MPO), both of which can directly cause tissue damage $[1,42]$. In fact, DNA, histones, NE and MPO are commonly used to identify NETs in experiments or human samples [1,43]. Though extracellular traps are most clearly observed with neutrophils supported by a numerous amount of studies, similar structures were found to be released from other cell types including macrophages (METs), mast cells (MCETs), eosinophils (EETs) and even B- and T-cells [44-46]. 


\section{Active release}

Besides the different types of cell death, DAMPs can be released from living or normal cells as well. Many types of DAMPs cannot be released by the typical secretory pathway for proteins consisting of endoplasmic reticulum (ER) and Golgi apparatus as they are nucleotides or proteins without a signal peptide. Well studied carriers of DAMPs during their active release are secretory lysosomes and exosomes, both of which are typically secreted by exocytosis. Even though exocytosis is a regular process even under steady state, increased DAMP release has been observed in this mechanism under stress condition [47-53].

\section{Secretory lysosomes}

Some DAMPs have been reported to be released via lysosomal exocytosis [36, 47, 50, 51]. The lysosomal import of soluble contents such as hydrolases, which unlike membrane proteins lack sorting signals, is at least in part mediated by their modification with mannose-6-phosphate, which is recognized by mannose-6-phosphate receptor proteins to be loaded into lysosomes [54]. Lysosome secretion can be initiated by the stimulation of cell-surface receptors leading to the increase of intracellular $\mathrm{Ca}^{2+}$, which is detected by synaptotagmin (Syt). Syt mobilizes the lysosomes toward microtubule organizing center (MTOC), where the lysosomes associate with a kinesin motor. The motor further transports lysosomes near to the site of secretion, where the lysosomes use actin-based movement to travel to the docking site. Docking and fusion of the lysosomes with the plasma membrane are mediated by RABs and SNARE complexes, respectively [54]. Lysosomal secretion is typical of cells that are stressed and has been identified as one of the release mechanisms of HMGB1, ATP and eCIRP [36, 47, 50, 51].

\section{Exosomes}

Another mechanism by which DAMPs are released from living cells is via exosomes. Exosomes are extracellular vesicles containing proteins, lipids and nucleic acids to facilitate intercellular communication. Intraluminal vesicles (ILV) — the future exosomes - are formed by inward budding of endosomes to form multivesicular bodies (MVBs) [55]. A well-studied regulator of exosome biogenesis and of the import of its components is the Endosomal Sorting Complex Required for Transport (ESCRT), which works along with its related proteins VPS4, VTA1, ALIX [56, 57]. MVBs are transported to the plasma membrane via interaction with actin, cortactin, microtubule skeleton and RAB proteins [56]. Similar to secretory lysosomes, the MVB docking to the plasma membrane and fusion with it are facilitated by RAB proteins and SNARE complexes, respectively [57].
A large number of DAMPs have been found to be present inside or on the surface of exosomes as we have previously reviewed [58]. Exosomal DAMPs include but are not limited to HMGB1, ATP, histones, HSPs, exRNAs and cfDNA [48, $52,53,58-61]$.

\section{DAMP receptors}

Released DAMPs are recognized by their corresponding receptors including, but not limited to, Toll-like receptors (TLRs), NOD-like receptors (NLRs), receptor for advanced glycation end products (RAGE), triggering receptors expressed on myeloid cells (TREMs) and P2X receptors (P2XRs). Activation of DAMP receptors induces inflammatory response such as cytokine and chemokine production among other effector functions [62]. In addition, the stimulation of the receptors by DAMPs in turn causes DAMP release. For instance, HMGB1 has shown to be released upon NLR and TLR stimulation both passively by cell death and actively by exocytosis [48, 62]. Even though not all the studies about the preceding receptors and DAMP release actually used DAMPs as stimuli of the receptors in the experimental design, theoretically DAMPs may also cause the similar phenomena by acting on the receptors to induce the downstream signaling pathways. Above all, there do exist studies showing that ATP activates $\mathrm{P} 2 \mathrm{X} 7$ to induce HMGB1 release via the activation of inflammasome as well as p38MAPK/NF- $\mathrm{kB}$ signaling accompanied by ROS accumulation $[63,64]$.

\section{Characteristics and release mechanisms for specific DAMPs}

\section{HMGB1}

HMGB1 is a nuclear protein capable of binding chromosomal DNA to fulfill its nuclear functions in stabilizing nucleosomal structure and stability and regulating gene expression. As a prototypical DAMP, HMGB1 can be passively released by somatic cells undergoing cytoplasmic membrane destruction due to accidental necrosis or regulated cell death processes such as necroptosis, pyroptosis ferroptosis, or apoptosis [16, 23, 27, 34-36, 41, 65, 66]. In addition, HMGB 1 can also be actively secreted by innate immune cells in response to microbial infections. Lacking a leader peptide sequence, HMGB1 cannot be actively secreted through classical ER - Golgi exocytotic pathways. Instead, its active secretion is generally regulated by two signaling steps. The first step, translocation from the nucleus to cytoplasm, is induced by JAK/STAT-1 mediated acetylation of the nuclear localization sequences (NLS) within HMGB1 [39]. The translocation has also shown to be 
mediated by the interaction with HSP90AA1 (heat shock protein 90 alpha family class A member 1) and XPO1 [49]. The second step, secretion from the cytoplasm to the extracellular space, is dependent on the activation of inflammasomes and caspases as described earlier under pyroptosis [34-36]. The latter step is at least partially controlled by double-stranded RNA-activated protein kinase R (PKR) [67]. A recent study has shown that unlike IL-1ß the release of HMGB1 by inflammasome activation was not through GSDMD pore but due to membrane rupture even though it was indeed GSDMD dependent [38]. In addition to its release by pyroptotic cells, HMGB1 is also released by living cells by exocytosis of lysosomes or exosomes [36, 47, 48]. HMGB1 has been found inside secretory lysosomes and it has also reported to be released via exosomes in a TLR4 and caspase-11/gasdermin D dependent manner [36, 47, 48]. Another study has shown that HMGB1 secretion was induced by autophagy machinery and MVB formation, resulting in its release in the form of exosomes [49].

\section{ATP}

ATP is a nucleotide essential for cellular metabolism, however extracellular ATP works as a DAMP via P2XRs [1]. Dosch et al. recently reviewed the mechanisms of ATP release intensively [68]. Like other DAMPs, ATP can be released passively from damaged tissues and dying cells undergoing necrosis or apoptosis and pyroptosis [17, 29, 37, 68]. Mainly two mechanisms have been reported for active ATP release; exocytosis and channel pores. ATP has been detected inside secretory lysosomes and vesicles and it has been proposed to be released via exocytosis [50, 61, 69]. The vesicular nucleotide transporter (VNUT) protein is responsible for the vesicular storage of ATP [69]. ATP is also exported via the pores of connexin and pannexin hemichannels [37, 68, 70, 71]. Connexins and pannexins are both four-pass transmembrane proteins and are represented by connexin 43 and pannexin 1, respectively. While they are likely to be closed under homeostatic conditions, connexin and pannexin hemichannels open in response to various triggers [68]. The state of connexin 43 is regulated by intracellular calcium concentrations, positive cell membrane voltage changes and phosphorylation of its serine residues. Moreover, the expression of connexin 43 is induced by TLR stimulation followed by ERK/AP-1 signaling [68, 72]. Pannexin 1 channels are opened by mechanical stress, increased intracellular calcium or extracellular potassium, and the cleavage of the $\mathrm{C}$-terminal tail of pannexin-1 proteins induced by activated caspase-3/caspase- 7 and caspase- 11 during apoptosis and pyroptosis, respectively $[37,68]$. To induce a more effective localized release of ATP, pannexin 1 has been reported to translocate to the leading edge of polarized neutrophils or the immune synapse of T-cells $[71,73,74]$.

\section{eCIRP}

CIRP is an RNA chaperone protein which plays a role in the regulation of a variety of cellular stress responses. Extracellular CIRP (eCIRP) is a DAMP that perpetuates inflammation and contributes to various diseases [5]. An in vitro study showed that passive release by necrosis might not be a major source of eCIRP, although passive release is a likely important source of CIRP in conditions such as trauma, ischemiareperfusion injury, and sepsis $[5,51]$. Extracellular traps have also shown to be sources of eCIRP [42]. Like HMGB1, CIRP does not contain a signal peptide, thus its release is unlikely to be mediated by ER-Golgi dependent classical pathway. The nuclear to cytoplasm translocation of CIRP requires post-translational modifications such as methylation and phosphorylation $[75,76]$. The phosphorylation of CIRP was shown to be mediated by GSK $3 \beta$ and casein kinase II (CK2) [76]. CIRP migrates from the nucleus to cytoplasmic stress granules under certain conditions such as oxidative stress, ER stress, hyperosmotic, and heat shock [75]. Stress granules are RNA-protein complexes that assemble when cells undergo polysome disassembly effectively interrupt protein translation in response to the conditions such as those above. When the offending condition is controlled, stress granules disassemble and translation resumes [77-79]. Stress granules interact with the inflammasome pathway as they inhibit NLRP3 inflammasome assembly by sequestering DDX3X protein [32]. Studies have shown stress granule proteins can be released via extracellular vesicles such as exosomes, though it's still not clear whether CIRP can be released through the same pathway [80-82]. Exocytosis of secretory lysosomes is also likely to contribute to CIRP release as a study showed CIRP was enriched at the lysosomal compartment of macrophages subjected to hypoxia [51].

\section{Histones}

Histones are components of chromatin in the nucleus together with DNA, but can act as DAMPs by binding to PRRs once they are released to the extracellular space [1]. Histones can be released passively by necrosis like other DAMPs $[1,18]$. Upon apoptotic signaling, core histones (H2A, H2B, H3, and H4) and a link histone (H1) undergo post-translational modifications (e.g., H2B phosphorylation at serine 14, H2B acetylation at lysine 15, etc.), which have been reviewed in depth by Füllgrabe et al. [83]. During apoptosis, the modified histones separate from the genomic DNA and translocate to the cytoplasm. The histones protrude from the plasma membrane to be exposed at the cell surface and released to the extracellular space through a 
mechanism that has not yet been elucidated [26]. One of the main components of NETs, citrullinated histones, mediated by PAD4, are released by the NETosis mechanisms described earlier [1]. Histones can also be secreted actively from living cells via exosomal exocytosis. A study has shown that histones were present on the outer surface of exosomes released from LPS-challenged macrophages and interacted with TLR4 directly [52].

\section{HSPs}

HSPs are a family of molecular chaperones maintaining cellular homeostasis [84]. Extracellular HSPs act as DAMPs and correlate with the severity of several disorders such as sepsis and trauma $[85,86]$. HSPs can be released passively by necrosis $[19,53]$. HSPs lack a secretory signal, thus are unlikely to be released via ER-Golgi transportation. Proposed mechanisms for active HSP release include secretion via ATP-binding cassette $(\mathrm{ABC})$ transporter in the lysosomal pathway and via secretory granules $[53,87,88]$. Above all, the most accepted mechanism of HSP release is via extracellular vesicles. HSPs are found in two types of extracellular vesicles; exosomes and ectosomes [53]. While exosomes are released by the mechanism described in the earlier section, ectosomes are formed by the outward budding of plasma membrane in response to the increase of cytosolic free $\mathrm{Ca}^{2+}$ and are released directly to the extracellular space. Ectosomes are also called microvesicles or microparticles [89]. Different types of HSP family, such as HSP27, HSP60, HSP70 and HSP90, have been found to be released via extracellular vesicles as reviewed by Maio et al. [53]. While HSPs might be located within the vesicle lumen, multiple studies showed HSPs were at least in part present on the surface of the extracellular vesicles, allowing them to interact with surface receptors directly [90-94].

\section{exRNAs}

While some exRNAs are known to have beneficial effect, other act as DAMPs to aggravate inflammation [95]. Though exRNAs can be released by necrosis and apoptosis, they are regarded to be more stable when encapsulated within extracellular vesicles, typically in exosomes, to avoid degradation by RNases in biological fluids such as saliva, breast milk, blood, cerebrospinal fluid, follicular fluid, and urine $[22,30,60]$. The import of exRNAs, specifically miRNAs, to extracellular vesicles is mediated by the association with argonaute 2 (Ago2) regulated by KRAS-MEK-ERK signaling [96]. Other RNA binding proteins (RBPs), such as Y-box protein 1 (YBX1), hnRNPA2B1 and SYNCRIP (hnRNPQ), have also been reported to play a role in the sorting of miRNAs [97-100]. RNAs loaded to the extracellular vesicles are released via exocytosis to become exRNAs. RBPs not only mediate the import but also act as carriers of exRNA in the circulation even without the encapsulation by extracellular vesicles [101, 102]. It is still unknown whether RBP/ exRNA complexes in the blood are predominantly released passively by cell death or exported actively via an independent pathway.

\section{cfDNA}

DNA in the extracellular space can serve as a DAMP. Various types of cell death, such as necrosis, apoptosis, pyroptosis ferroptosis, and NETosis are known to release DNA $[1,10,20,26,30,33,40]$. Its mechanisms of release differ according to the pathogenic condition exemplified by NETosis in sepsis patients and necrotic cells in trauma patients [20]. A recent study showed that cellular senescence was a major determinant of cfDNA kinetics by negatively regulating its release. The elimination of senescent cells through apoptosis recovered cfDNA release [103]. In addition to the passive release by cell death, DNA can be released actively via extracellular vesicles, including exosomes and ectosomes [104]. A study suggests that majority of cfDNA in the blood is present in the form of exosomes, thus avoiding its degradation by nucleases [59]. Besides nuclear DNA, cell-free mitochondrial DNA (cf-mtDNA) also acts as a DAMP [105]. Cf-mtDNA was found in platelet-driven ectosomes along with phospholipase A2 (PLA2). PLA2 is a bactericidal enzyme found to digest the cellular membrane leading to the leakage of mtDNA into the extracellular space [106].

\section{Conclusions and future prospects}

DAMPs represented here by HMGB1, ATP, eCIRP, histones, HSPs, exRNAs and cfDNA can be released by several different active and passive mechanisms such as exocytosis of lysosomes/exosomes, necrosis/necroptosis, apoptosis, pyroptosis, ferroptosis, and extracellular traps. Some mechanisms such as necrosis are shared with more DAMPs, while others are relatively DAMP-specific, e.g., channel pores for ATP.

Despite the detailed mechanisms reviewed here, clearly a lot still remains to be elucidated. A more comprehensive understanding of the mechanisms DAMP release and their regulation will not only enable the design of new investigative tools but may also result in new potential therapeutic approaches to attenuate inflammation and tissue injury and thus to improve the outcomes of pathological conditions associated with excessive DAMP release.

Author contributions AM, MB designed, wrote and revised the review article. AM, MB reviewed the literature. MA, HW and PW reviewed 
and edited the paper. PW conceived the idea. MB, PW supervised the work.

Funding This study was supported by the National Institutes of Health (NIH) grants R35GM118337 and R01HL076179 (P.W.), R01AA028947 (P.W. and Philippe Marambaud), U01AI133655 (P.W. and M.B.), and R01GM129633 (M.A.).

Data availability Not applicable.

\section{Declarations}

Conflict of interest The authors declare no conflicts of interest.

Consent to participate Not applicable.

Consent for publication Not applicable.

Ethical approval Not applicable

Open Access This article is licensed under a Creative Commons Attribution 4.0 International License, which permits use, sharing, adaptation, distribution and reproduction in any medium or format, as long as you give appropriate credit to the original author(s) and the source, provide a link to the Creative Commons licence, and indicate if changes were made. The images or other third party material in this article are included in the article's Creative Commons licence, unless indicated otherwise in a credit line to the material. If material is not included in the article's Creative Commons licence and your intended use is not permitted by statutory regulation or exceeds the permitted use, you will need to obtain permission directly from the copyright holder. To view a copy of this licence, visit http://creativecommons.org/licenses/by/4.0/.

\section{References}

1. Denning NL, Aziz M, Gurien SD, Wang P (2019) DAMPs and NETs in Sepsis. Front Immunol 10:2536

2. Jounai N, Kobiyama K, Takeshita F, Ishii KJ (2012) Recognition of damage-associated molecular patterns related to nucleic acids during inflammation and vaccination. Front Cell Infect Microbiol 2:168

3. Calderwood SK, Gong J, Murshid A (2016) Extracellular HSPs: the complicated roles of extracellular HSPs in immunity. Front Immunol 7:159

4. Krysko O, Løve Aaes T, Bachert C, Vandenabeele P, Krysko DV (2013) Many faces of DAMPs in cancer therapy. Cell Death Dis 4:e631

5. Aziz M, Brenner M, Wang P (2019) Extracellular CIRP (eCIRP) and inflammation. J Leukoc Biol 106:133-146

6. Hernandez C, Huebener P, Schwabe RF (2016) Damage-associated molecular patterns in cancer: a double-edged sword. Oncogene 35:5931-5941

7. Roh JS, Sohn DH (2018) Damage-associated molecular patterns in inflammatory diseases. Immune Netw 18:e27

8. Land WG (2020) Use of DAMPs and SAMPs as therapeutic targets or therapeutics: a note of caution. Mol Diagn Ther 24:251-262

9. Honore PM, Hoste E, Molnár Z et al (2019) Cytokine removal in human septic shock: where are we and where are we going? Ann Intensive Care 9:56
10. Jahr S, Hentze H, Englisch $\mathrm{S}$ et al (2001) DNA fragments in the blood plasma of cancer patients: quantitations and evidence for their origin from apoptotic and necrotic cells. Cancer Res 61:1659-1665

11. Yang H, Wang H, Chavan SS, Andersson U (2015) High mobility group box protein 1 (HMGB1): the prototypical endogenous danger molecule. Mol Med 21(Suppl 1):S6-S12

12. Garg AD, Krysko DV, Verfaillie T et al (2012) A novel pathway combining calreticulin exposure and ATP secretion in immunogenic cancer cell death. EMBO J 31:1062-1079

13. Hayashi K, Nikolos F, Lee YC et al (2020) Tipping the immunostimulatory and inhibitory DAMP balance to harness immunogenic cell death. Nat Commun 11:6299

14. Choi ME, Price DR, Ryter SW, Choi AMK (2019) Necroptosis: a crucial pathogenic mediator of human disease. JCI Insight 4:e128834

15. Szabó C (2005) Mechanisms of cell necrosis. Crit Care Med 33:S530-S534

16. Scaffidi P, Misteli T, Bianchi ME (2002) Release of chromatin protein HMGB1 by necrotic cells triggers inflammation. Nature 418:191-195

17. Iyer SS, Pulskens WP, Sadler JJ et al (2009) Necrotic cells trigger a sterile inflammatory response through the Nlrp3 inflammasome. Proc Natl Acad Sci U S A 106:20388-20393

18. Allam R, Darisipudi MN, Tschopp J, Anders HJ (2013) Histones trigger sterile inflammation by activating the NLRP3 inflammasome. Eur J Immunol 43:3336-3342

19. Basu S, Binder RJ, Suto R, Anderson KM, Srivastava PK (2000) Necrotic but not apoptotic cell death releases heat shock proteins, which deliver a partial maturation signal to dendritic cells and activate the NF-kappa B pathway. Int Immunol 12:1539-1546

20. Jackson Chornenki NL, Coke R, Kwong AC et al (2019) Comparison of the source and prognostic utility of cfDNA in trauma and sepsis. Intensive Care Med Exp 7:29

21. Tielking K, Fischer S, Preissner KT, Vajkoczy P, Xu R (2019) Extracellular RNA in central nervous system pathologies. Front Mol Neurosci 12:254

22. Brentano F, Schorr O, Gay RE, Gay S, Kyburz D (2005) RNA released from necrotic synovial fluid cells activates rheumatoid arthritis synovial fibroblasts via Toll-like receptor 3. Arthritis Rheum 52:2656-2665

23. Kaczmarek A, Vandenabeele P, Krysko DV (2013) Necroptosis: the release of damage-associated molecular patterns and its physiological relevance. Immunity 38:209-223

24. Tang D, Kang R, Berghe TV, Vandenabeele P, Kroemer G (2019) The molecular machinery of regulated cell death. Cell Res 29:347-364

25. Montico B, Nigro A, Casolaro V, Dal Col J (2018) Immunogenic apoptosis as a novel tool for anticancer vaccine development. Int J Mol Sci 19

26. Radic M, Marion T, Monestier M (2004) Nucleosomes are exposed at the cell surface in apoptosis. J Immunol 172:6692-6700

27. Jiang W, Bell CW, Pisetsky DS (2007) The relationship between apoptosis and high-mobility group protein 1 release from murine macrophages stimulated with lipopolysaccharide or polyinosinic-polycytidylic acid. J Immunol 178:6495-6503

28. Chen R, Kang R, Fan XG, Tang D (2014) Release and activity of histone in diseases. Cell Death Dis 5:e1370

29. Elliott MR, Chekeni FB, Trampont PC et al (2009) Nucleotides released by apoptotic cells act as a find-me signal to promote phagocytic clearance. Nature 461:282-286

30. Reich CF, Pisetsky DS (2009) The content of DNA and RNA in microparticles released by Jurkat and HL-60 cells undergoing in vitro apoptosis. Exp Cell Res 315:760-768 
31. Shi J, Gao W, Shao F (2017) Pyroptosis: Gasdermin-mediated programmed necrotic cell death. Trends Biochem Sci 42:245-254

32. Samir P, Kesavardhana S, Patmore DM et al (2019) DDX3X acts as a live-or-die checkpoint in stressed cells by regulating NLRP3 inflammasome. Nature 573:590-594

33. Tan HY, Yong YK, Shankar EM et al (2016) Aberrant inflammasome activation characterizes tuberculosis-associated immune reconstitution inflammatory syndrome. J Immunol 196:4052-4063

34. Hou L, Yang Z, Wang Z et al (2018) NLRP3/ASC-mediated alveolar macrophage pyroptosis enhances HMGB1 secretion in acute lung injury induced by cardiopulmonary bypass. Lab Invest 98:1052-1064

35. Lamkanfi M, Sarkar A, Vande Walle L et al (2010) Inflammasome-dependent release of the alarmin HMGB1 in endotoxemia. J Immunol 185:4385-4392

36. Lu B, Wang C, Wang M et al (2014) Molecular mechanism and therapeutic modulation of high mobility group box 1 release and action: an updated review. Expert Rev Clin Immunol 10:713-727

37. Yang D, He Y, Muñoz-Planillo R, Liu Q, Núñez G (2015) Caspase-11 requires the pannexin-1 channel and the purinergic $\mathrm{P} 2 \times 7$ pore to mediate pyroptosis and endotoxic shock. Immunity 43:923-932

38. Volchuk A, Ye A, Chi L, Steinberg BE, Goldenberg NM (2020) Indirect regulation of HMGB1 release by gasdermin D. Nat Commun 11:4561

39. Lu B, Antoine DJ, Kwan K et al (2014) JAK/STAT1 signaling promotes HMGB1 hyperacetylation and nuclear translocation. Proc Natl Acad Sci U S A 111:3068-3073

40. Tang D, Chen X, Kang R, Kroemer G (2020) Ferroptosis: molecular mechanisms and health implications. Cell Res. https://doi. org/10.1038/s41422-020-00441-1

41. Wen Q, Liu J, Kang R, Zhou B, Tang D (2019) The release and activity of HMGB1 in ferroptosis. Biochem Biophys Res Commun 510:278-283

42. Linders J, Madhi R, Rahman M et al (2020) Extracellular coldinducible RNA-binding protein regulates neutrophil extracellular trap formation and tissue damage in acute pancreatitis. Lab Invest 100:1618-1630

43. Mutua V, Gershwin LJ (2020) A review of neutrophil extracellular traps (NETs) in disease: Potential anti-nets therapeutics. Clin Rev Allergy Immunol Aug 1:1-18. https://doi.org/10.1007/ s12016-020-08804-7

44. Ingelsson B, Söderberg D, Strid T et al (2018) Lymphocytes eject interferogenic mitochondrial DNA webs in response to $\mathrm{CpG}$ and non-CpG oligodeoxynucleotides of class C. Proc Natl Acad Sci U S A 115:E478-E487

45. Rocha Arrieta YC, Rojas M, Vasquez G, Lopez J (2017) The lymphocytes stimulation induced DNA release, a phenomenon similar to NETosis. Scand J Immunol 86:229-238

46. Pertiwi KR, de Boer OJ, Mackaaij C et al (2019) Extracellular traps derived from macrophages, mast cells, eosinophils and neutrophils are generated in a time-dependent manner during atherothrombosis. J Pathol 247:505-512

47. Gardella S, Andrei C, Ferrera D et al (2002) The nuclear protein HMGB1 is secreted by monocytes via a non-classical, vesiclemediated secretory pathway. EMBO Rep 3:995-1001

48. Li W, Deng M, Loughran PA et al (2020) LPS induces active HMGB1 release from hepatocytes into exosomes through the coordinated activities of TLR4 and caspase-11/GSDMD signaling. Front Immunol 11:229

49. Kim YH, Kwak MS, Lee B et al (2020) Secretory autophagy machinery and vesicular trafficking are involved in HMGB1 secretion. Autophagy 5:1-18
50. Zhang Z, Chen G, Zhou W et al (2007) Regulated ATP release from astrocytes through lysosome exocytosis. Nat Cell Biol 9:945-953

51. Qiang X, Yang WL, Wu R et al (2013) Cold-inducible RNAbinding protein (CIRP) triggers inflammatory responses in hemorrhagic shock and sepsis. Nat Med 19:1489-1495

52. Nair RR, Mazza D, Brambilla F, Gorzanelli A, Agresti A, Bianchi ME (2018) LPS-challenged macrophages release microvesicles coated with histones. Front Immunol 9:1463

53. De Maio A, Vazquez D (2013) Extracellular heat shock proteins: a new location, a new function. Shock 40:239-246

54. Blott EJ, Griffiths GM (2002) Secretory lysosomes. Nat Rev Mol Cell Biol 3:122-131

55. Kalluri R, LeBleu VS (2020) The biology, function, and biomedical applications of exosomes. Science 367:eaau6977

56. Tschuschke M, Kocherova I, Bryja A et al (2020) Inclusion biogenesis, methods of isolation and clinical application of human cellular exosomes. J Clin Med 9:436

57. Colombo M, Raposo G, Théry C (2014) Biogenesis, secretion, and intercellular interactions of exosomes and other extracellular vesicles. Annu Rev Cell Dev Biol 30:255-289

58. Murao A, Brenner M, Aziz M, Wang P (2020) Exosomes in sepsis. Front Immunol 11:2140

59. Fernando MR, Jiang C, Krzyzanowski GD, Ryan WL (2017) New evidence that a large proportion of human blood plasma cell-free DNA is localized in exosomes. PLoS One 12:e0183915

60. Sadik N, Cruz L, Gurtner A et al (2018) Extracellular RNAs: a new awareness of old perspectives. Methods Mol Biol 1740:1-15

61. Sakaki H, Tsukimoto M, Harada H, Moriyama Y, Kojima S (2013) Autocrine regulation of macrophage activation via exocytosis of ATP and activation of P2Y11 receptor. PLoS ONE 8:e59778

62. Gong T, Liu L, Jiang W, Zhou R (2020) DAMP-sensing receptors in sterile inflammation and inflammatory diseases. Nat Rev Immunol 20:95-112

63. Hiramoto S, Tsubota M, Yamaguchi K et al (2020) Cystitisrelated bladder pain involves ATP-dependent HMGB1 release from macrophages and its downstream $\mathrm{H}_{2} \mathrm{~S} / \mathrm{Ca}_{\mathrm{v}} 3.2$ signaling in mice. Cells 9:1748

64. Johnson L, Atanasova KR, Bui PQ et al (2015) Porphyromonas gingivalis attenuates ATP-mediated inflammasome activation and HMGB1 release through expression of a nucleoside-diphosphate kinase. Microbes Infect 17:369-377

65. Nakano H, Murai S, Yamaguchi Y, Shirasaki Y, Nakabayashi O, Yamazaki S (2019) Development of novel methods that monitor necroptosis and the release of DAMPs at the single cell resolution. Cell Stress 3:66-69

66. Simpson J, Loh Z, Ullah MA et al (2020) Respiratory syncytial virus infection promotes necroptosis and HMGB1 release by airway epithelial cells. Am J Respir Crit Care Med 201:1358-1371

67. Lu B, Nakamura T, Inouye K et al (2012) Novel role of PKR in inflammasome activation and HMGB1 release. Nature 488:670-674

68. Dosch M, Gerber J, Jebbawi F, Beldi G (2018) Mechanisms of ATP release by inflammatory cells. Int J Mol Sci 19:1222

69. Sawada K, Echigo N, Juge N et al (2008) Identification of a vesicular nucleotide transporter. Proc Natl Acad Sci USA 105:5683-5686

70. Kang J, Kang N, Lovatt D et al (2008) Connexin 43 hemichannels are permeable to ATP. J Neurosci 28:4702-4711

71. Woehrle T, Yip L, Elkhal A et al (2010) Pannexin-1 hemichannel-mediated ATP release together with $\mathrm{P} 2 \times 1$ and $\mathrm{P} 2 \times 4$ receptors regulate $\mathrm{T}$-cell activation at the immune synapse. Blood $116: 3475-3484$ 
72. Qin J, Zhang G, Zhang X et al (2016) TLR-activated gap junction channels protect mice against bacterial infection through extracellular UDP release. J Immunol 196:1790-1798

73. Junger WG (2011) Immune cell regulation by autocrine purinergic signalling. Nat Rev Immunol 11:201-212

74. Chen Y, Yao Y, Sumi Y et al (2010) Purinergic signaling: a fundamental mechanism in neutrophil activation. Sci Signal 3:ra45

75. De Leeuw F, Zhang T, Wauquier C, Huez G, Kruys V, Gueydan C (2007) The cold-inducible RNA-binding protein migrates from the nucleus to cytoplasmic stress granules by a methylationdependent mechanism and acts as a translational repressor. Exp Cell Res 313:4130-4144

76. Yang R, Zhan M, Nalabothula NR, Yang Q, Indig FE, Carrier F (2010) Functional significance for a heterogenous ribonucleoprotein A18 signature RNA motif in the 3'-untranslated region of ataxia telangiectasia mutated and Rad3-related (ATR) transcript. J Biol Chem 285:8887-8893

77. Van Treeck B, Protter DSW, Matheny T, Khong A, Link CD, Parker R (2018) RNA self-assembly contributes to stress granule formation and defining the stress granule transcriptome. Proc Natl Acad Sci U S A 115:2734-2739

78. Jeong SG, Ohn T, Jang CH, Vijayakumar K, Cho GW (2020) The role of stress granules in the neuronal differentiation of stem cells. Mol Cells 43:848-855

79. Hofmann S, Kedersha N, Anderson P, Ivanov P (2020) Molecular mechanisms of stress granule assembly and disassembly. Biochim Biophys Acta Mol Cell Res 1868:118876

80. de Jong OG, Verhaar MC, Chen Y et al (2012) Cellular stress conditions are reflected in the protein and RNA content of endothelial cell-derived exosomes. J Extracell Vesicles. https:// doi.org/10.3402/jev.v1i0.18396

81. Statello L, Maugeri M, Garre E et al (2018) Identification of RNA-binding proteins in exosomes capable of interacting with different types of RNA: RBP-facilitated transport of RNAs into exosomes. PLoS ONE 13:e0195969

82. Vassileff N, Vella LJ, Rajapaksha H et al (2020) Revealing the proteome of motor cortex derived extracellular vesicles isolated from amyotrophic lateral sclerosis human postmortem tissues. Cells 9:1709

83. Füllgrabe J, Hajji N, Joseph B (2010) Cracking the death code: apoptosis-related histone modifications. Cell Death Differ 17:1238-1243

84. Creagh EM, Sheehan D, Cotter TG (2000) Heat shock proteins-modulators of apoptosis in tumour cells. Leukemia 14:1161-1173

85. Fitrolaki MD, Dimitriou H, Venihaki M, Katrinaki M, Ilia S, Briassoulis G (2016) Increased extracellular heat shock protein $90 \alpha$ in severe sepsis and SIRS associated with multiple organ failure and related to acute inflammatory-metabolic stress response in children. Medicine 95:e4651

86. Haider T, Simader E, Glück O et al (2019) Systemic release of heat-shock protein 27 and 70 following severe trauma. Sci Rep 9:9595

87. Mambula SS, Calderwood SK (2006) Heat shock protein 70 is secreted from tumor cells by a nonclassical pathway involving lysosomal endosomes. J Immunol 177:7849-7857

88. Evdonin AL, Martynova MG, Bystrova OA, Guzhova IV, Margulis BA, Medvedeva ND (2006) The release of Hsp70 from A431 carcinoma cells is mediated by secretory-like granules. Eur J Cell Biol 85:443-455

89. Cocucci E, Meldolesi J (2011) Ectosomes. Curr Biol 21:R940-R941

90. Vega VL, Rodríguez-Silva M, Frey T et al (2008) Hsp70 translocates into the plasma membrane after stress and is released into the extracellular environment in a membrane-associated form that activates macrophages. J Immunol 180:4299-4307
91. Gastpar R, Gehrmann M, Bausero MA et al (2005) Heat shock protein 70 surface-positive tumor exosomes stimulate migratory and cytolytic activity of natural killer cells. Cancer Res 65:5238-5247

92. Chalmin F, Ladoire S, Mignot G et al (2010) Membrane-associated Hsp72 from tumor-derived exosomes mediates STAT3dependent immunosuppressive function of mouse and human myeloid-derived suppressor cells. J Clin Invest 120:457-471

93. Gupta S, Knowlton AA (2007) HSP60 trafficking in adult cardiac myocytes: role of the exosomal pathway. Am J Physiol Heart Circ Physiol 292:H3052-H3056

94. Merendino AM, Bucchieri F, Campanella C et al (2010) Hsp60 is actively secreted by human tumor cells. PLoS ONE 5:e9247

95. Roers A, Hiller B, Hornung V (2016) Recognition of endogenous nucleic acids by the innate immune system. Immunity 44:739-754

96. McKenzie AJ, Hoshino D, Hong NH et al (2016) KRASMEK signaling controls Ago2 sorting into exosomes. Cell Rep 15:978-987

97. Li K, Rodosthenous RS, Kashanchi F et al (2018) Advances, challenges, and opportunities in extracellular RNA biology: insights from the NIH exRNA Strategic Workshop. JCI Insight 3

98. Shurtleff MJ, Temoche-Diaz MM, Karfilis KV, Ri S, Schekman $\mathrm{R}$ (2016) Y-box protein 1 is required to sort microRNAs into exosomes in cells and in a cell-free reaction. Elife 5

99. Villarroya-Beltri C, Gutiérrez-Vázquez C, Sánchez-Cabo F et al (2013) Sumoylated hnRNPA2B1 controls the sorting of miRNAs into exosomes through binding to specific motifs. Nat Commun 4:2980

100. Santangelo L, Giurato G, Cicchini C et al (2016) The RNA-binding protein SYNCRIP is a component of the hepatocyte exosomal machinery controlling microRNA sorting. Cell Rep 17:799-808

101. Arroyo JD, Chevillet JR, Kroh EM et al (2011) Argonaute2 complexes carry a population of circulating microRNAs independent of vesicles in human plasma. Proc Natl Acad Sci U S A 108:5003-5008

102. Turchinovich A, Weiz L, Langheinz A, Burwinkel B (2011) Characterization of extracellular circulating microRNA. Nucleic Acids Res 39:7223-7233

103. Rostami A, Lambie M, Yu CW, Stambolic V, Waldron JN, Bratman SV (2020) Senescence, necrosis, and apoptosis govern circulating cell-free DNA release kinetics. Cell Rep 31:107830

104. Duvvuri B, Lood C (2019) Cell-Free DNA as a biomarker in autoimmune rheumatic diseases. Front Immunol 10:502

105. Krysko DV, Agostinis P, Krysko O et al (2011) Emerging role of damage-associated molecular patterns derived from mitochondria in inflammation. Trends Immunol 32:157-164

106. Boudreau LH, Duchez AC, Cloutier N et al (2014) Platelets release mitochondria serving as substrate for bactericidal group IIA-secreted phospholipase A2 to promote inflammation. Blood 124:2173-2183

107. Wang Z, Yang C, Li L et al (2020) Tumor-derived HMGB1 induces CD62L. Oncogenesis 9:82

108. Sumi Y, Woehrle T, Chen Y et al (2014) Plasma ATP is required for neutrophil activation in a mouse sepsis model. Shock 42:142-147

109. Furuya K, Tan JJ, Boudreault F, Sokabe M, Berthiaume Y, Grygorczyk R (2016) Real-time imaging of inflation-induced ATP release in the ex vivo rat lung. Am J Physiol Lung Cell Mol Physiol 311:L956-L969

110. Kirby BS, Sparks MA, Lazarowski ER, Lopez Domowicz DA, Zhu H, McMahon TJ (2021) Pannexin 1 channels control the hemodynamic response to hypoxia by regulating $\mathrm{O}_{2}$-sensitive extracellular ATP in blood. Am J Physiol Heart Circ Physiol. https://doi.org/10.1152/ajpheart.00651.20202020 
111. Kitajima N, Takikawa K, Sekiya $\mathrm{H}$ et al (2020) Real-time in vivo imaging of extracellular ATP in the brain with a hybrid-type fluorescent sensor. Elife 9:e57544

112. Ito T, Nakahara M, Masuda $Y$ et al (2018) Circulating histone H3 levels are increased in septic mice in a neutrophil-dependent manner: preclinical evaluation of a novel sandwich ELISA for histone H3. J Intensive Care 6:79

113. Abrams ST, Zhang N, Manson J et al (2013) Circulating histones are mediators of trauma-associated lung injury. Am J Respir Crit Care Med 187:160-169

114. Wen Z, Liu Y, Li F et al (2013) Circulating histones exacerbate inflammation in mice with acute liver failure. J Cell Biochem 114:2384-2391

115. Nakazawa D, Kumar SV, Marschner J et al (2017) Histones and neutrophil extracellular traps enhance tubular necrosis and remote organ injury in ischemic AKI. J Am Soc Nephrol 28:1753-1768

116. Bosmann M, Grailer JJ, Ruemmler R et al (2013) Extracellular histones are essential effectors of C5aR- and C5L2-mediated tissue damage and inflammation in acute lung injury. FASEB J 27:5010-5021

117. Tsai TN, Lee TY, Liu MS et al (2015) Release of endogenous heat shock protein 72 on the survival of sepsis in rats. J Surg Res 198:165-174

118. Singleton KD, Wischmeyer PE (2006) Effects of HSP70.1/3 gene knockout on acute respiratory distress syndrome and the inflammatory response following sepsis. Am J Physiol Lung Cell Mol Physiol 290:L956-L961

119. Zhang G, Liu Z, Ding H et al (2017) Tumor induces muscle wasting in mice through releasing extracellular Hsp70 and Hsp90. Nat Commun 8:589

120. Gurien SD, Aziz M, Jin $\mathrm{H}$ et al (2020) Extracellular microRNA 130b-3p inhibits eCIRP-induced inflammation. EMBO Rep 21:e48075

121. Zou L, Feng Y, Xu G, Jian W, Chao W (2016) Splenic RNA and MicroRNA mimics promote complement factor B production and alternative pathway activation via innate immune signaling. J Immunol 196:2788-2798

122. Jiang K, Yang J, Guo S, Zhao G, Wu H, Deng G (2019) Peripheral circulating exosome-mediated delivery of miR-155 as a novel mechanism for acute lung inflammation. Mol Ther 27:1758-1771

123. Lv LL, Feng Y, Wu M et al (2020) Exosomal miRNA-19b-3p of tubular epithelial cells promotes M1 macrophage activation in kidney injury. Cell Death Differ 27:210-226

124. Bala S, Petrasek J, Mundkur S et al (2012) Circulating microRNAs in exosomes indicate hepatocyte injury and inflammation in alcoholic, drug-induced, and inflammatory liver diseases. Hepatology 56:1946-1957

125. Hamaguchi S, Akeda Y, Yamamoto $N$ et al (2015) Origin of circulating free DNA in sepsis: analysis of the CLP mouse model. Mediators Inflamm 2015:614518

126. Gruenbaum BF, Boyko M, Delgado B et al (2013) Cell-free DNA as a potential marker to predict carbon tetrachloride-induced acute liver injury in rats. Hepatol Int 7:721-727

127. Homolová J, Janovičová L, Konečná B et al (2020) Plasma concentrations of extracellular DNA in acute kidney injury. Diagnostics (Basel) 10:152

128. Khosrotehrani K, Wataganara T, Bianchi DW, Johnson KL (2004) Fetal cell-free DNA circulates in the plasma of pregnant mice: relevance for animal models of fetomaternal trafficking. Hum Reprod 19:2460-2464

129. Wei L, Xie L, Wang X et al (2018) Circulating tumor DNA measurement provides reliable mutation detection in mice with human lung cancer xenografts. Lab Invest 98:935-946

Publisher's Note Springer Nature remains neutral with regard to jurisdictional claims in published maps and institutional affiliations. 